\title{
Baryon structure in AdS/QCD
}

\author{
Valery E. Lyubovitskij ${ }^{* \dagger}$ \\ Institut für Theoretische Physik, Universität Tübingen, \\ Kepler Center for Astro and Particle Physics, \\ Auf der Morgenstelle 14, D-72076 Tübingen, Germany \\ E-mail: valeri.lyubovitskijeuni-tuebingen.de
}

\section{Thomas Gutsche}

Institut für Theoretische Physik, Universität Tübingen,

Kepler Center for Astro and Particle Physics,

Auf der Morgenstelle 14, D-72076 Tübingen, Germany

E-mail: thomas.gutscheduni-tuebingen.de

\section{Ivan Schmidt}

Departamento de Física y Centro Científico Tecnológico de Valparaíso (CCTVal), Universidad Técnica Federico Santa María, Casilla 110-V, Valparaíso, Chile

E-mail: ivan.schmidt@usm.cl

\section{Alfredo Vega}

Departamento de Física y Centro Científico Tecnológico de Valparaíso (CCTVal), Universidad Técnica Federico Santa María, Casilla 110-V, Valparaíso, Chile

E-mail: alfredo.vega@usm.cl

We perform a detailed analysis of nucleon electromagnetic and axial form factors in a holographic soft-wall model. Our approach is based on an action which describes hadrons with broken conformal invariance and incorporates confinement through the presence of a background dilaton field. For $N_{c}=3$ we describe the nucleon structure in a superposition of a three valence quark state with high Fock states including an adjustable number of partons (quarks, antiquarks and gluons) via studying the dynamics of 5D fermion fields of different scaling dimension in anti-de Sitter (ADS) space. According to the gauge/gravity duality the 5D fermion fields of different scaling dimension correspond to the Fock state components with a specific number of partons. In the present application we restrict to the contribution of 3, 4 and 5 parton components in the nucleon Fock state. With a minimal number of free parameters (dilaton scale parameter, mixing parameters of partial contributions of Fock states, coupling constants in the effective Lagrangian) we achieve a reasonable agreement with data for the nucleon form factors.

XXI International Baldin Seminar on High Energy Physics Problems,

September 10-15, 2012

JINR, Dubna, Russia

\footnotetext{
* Speaker.

${ }^{\dagger}$ On leave of absence from Department of Physics, Tomsk State University, 634050 Tomsk, Russia
} 


\section{Introduction}

Based on the gauge/gravity duality [1], a class of AdS/QCD approaches which model QCD by using methods of extra-dimensional field theories formulated in anti-de Sitter (AdS) space, was recently successfully developed for describing the phenomenology of hadronic properties (for a recent review see e.g. [目]). One of the popular formalisms of this kind is the "soft-wall" model [3][24], which uses a soft infrared (IR) cutoff in the fifth dimension. This procedure can be introduced in the following ways: i) as a background field (dilaton) in the overall exponential of the action ("dilaton" soft-wall model), ii) in the warping factor of the AdS metric ("metric" soft-wall model), iii) in the effective potential of the action. In Ref. [24] we showed that these three ways of proceeding are equivalent to each other via a redefinition of the bulk fields and by inclusion of extra effective potentials in the action. In our opinion, the "dilaton" form of the soft-wall model is more convenient in performing the calculations.

Applications of the soft-wall model to baryon physics have been worked out in Refs. [9, 18, 15, 22, 20, 24, 25, where the mass spectrum of light and heavy baryons, and electromagnetic and gravitational form factors have been calculated. We should stress that during last years significant progress in understanding of baryon structure using methods of AdS/QCD has been achieved [20, 26, 27, 28, 29, 30, 31, 32]. In particular, different types of hard-wall models have been suggested and developed in Refs. [20, 26, 27, 28]. Solitonic approaches, where stable solitons arise from an effective mesonic action which are 5D analogues of 4D Skyrmions, have been suggested in Refs. [29]. Direct derivations of holographic solitonic approaches for baryons from string theories have been proposed in Refs. [30, 31]. In reference to the 5D soliton AdS/QCD models developed in Refs. [29, 30, 31] we view our approach as an effective or phenomenological framework describing baryons in terms of fermion fields. As stressed in Ref. [27] this is not in contradiction with basic principles of QCD, because in 4D QCD the baryons can be described as skyrmions of the chiral meson Lagrangian or equivalently in terms of separate fermion fields coupled to mesons. In our approach the fermion bulk fields are characterized by the 5D mass $\mu$ (scaling dimension), which is holographically dual to $N$ - the number of partons in baryons. Both quantities scale in the large $N_{c}$ expansion as $\mu \sim N \sim N_{c}$, which means that the baryon is a bound state of $N_{c}$ quarks. This is consistent with large $N_{c}$ QCD. On the other hand, keeping in mind that in QCD the number of colors is equal $N_{c}=3$, we in physical applications identify the AdS fermion field of lowest dimension with the baryons containing three quarks. We do not restrict to the three-valence quark picture of baryons and also include higher Fock states involving nonvalence degrees of freedom. The latter are dual to the AdS fermion fields of higher dimension.

Here we present a detailed analysis of the nucleon electromagnetic form factors in a holographic soft-wall model considering the inclusion of higher-dimensional fermion fields. Thus highFock state contributions are holographically incorporated in the nucleon. This novel approach is based on an action which describes hadrons with broken conformal invariance and which incorporates confinement through the presence of a background dilaton field. Notice that the role of higher Fock components in the pion, in the context of holographic QCD, was considered before in Refs. [19], 33]. In particular, two Fock components $(q \bar{q}$ and $q \bar{q} q \bar{q})$ were included in the expansion of the pion wave function, which was in turn used in the calculation of pion electromagnetic and $\gamma \gamma^{*} \pi^{0}$ transition form factors. It was argued that the components containing gluons (e.g. $q \bar{q} g$ ) are 
absent in the confinement potential.

In our framework for $N_{c}=3$ nucleons are considered as a superposition of three valence quark states and high Fock states including an adjustable number of partons (quarks, antiquarks and gluons) by studying the dynamics of the 5D fermion fields of different scaling dimension in antide Sitter (ADS) space. According to the gauge/gravity duality the 5D fermion fields of different scaling dimension correspond to Fock state components with a specific number of partons. We can sum the bulk fermion actions with an adjustable 5D fermion mass, which is related to the scaling dimension (or the number of partons in the nucleon). This action is consistent with $C_{-}, P-$ and $T-$ invariance. Also, electromagnetic gauge invariance is fulfilled. Therefore, the main advantage of our approach is that it allows to include dynamically any adjustable number of higher Fock states in the nucleon. For this first time we restrict ourselves to the contribution of 3, 4 and 5 parton components in the nucleon Fock state.

\section{Approach}

We consider the propagation of a fermion field $\Psi(x, z)$ with spin $J=1 / 2$ in 5-dimensional AdS space, which contains the contributions of different twist-dimensions. In the language of the AdS/QCD dictionary it corresponds to the inclusion of the three-quark and higher-parton states in the nucleon. For this first time we restrict ourselves to the contribution of $3 q, 3 q+g, 3 q+q \bar{q}$ and $3 q+2 g$ Fock states, where $q, \bar{q}$ and $g$ denote quark, antiquark and gluon, respectively.

The AdS metric is specified by

$$
d s^{2}=g_{M N} d x^{M} d x^{N}=e^{2 A(z)}\left(\eta_{\mu v} d x^{\mu} d x^{v}-d z^{2}\right), \quad \eta_{\mu \nu}=\operatorname{diag}(1,-1,-1,-1,-1),
$$

where $M$ and $N=0,1, \cdots, 4$ are the space-time (base manifold) indices, $a=(\mu, z)$ and $b=(v, z)$ are the local Lorentz (tangent) indices, and $g_{M N}$ and $\eta_{a b}$ are curved and flat metric tensors, which are related by the vielbein $\varepsilon_{M}^{a}(z)=e^{A(z)} \delta_{M}^{a}$ as $g_{M N}=\varepsilon_{M}^{a} \varepsilon_{N}^{b} \eta_{a b}$. Here $z$ is the holographic coordinate, $R$ is the AdS radius, and $g=\left|\operatorname{det} g_{M N}\right|=e^{10 A(z)}$. In the following we restrict ourselves to a conformalinvariant metric with $A(z)=\log (R / z)$.

The main idea for describing the nucleon in AdS/QCD is based on the correspondence (see detailed discussion in Refs. [32, 27, 28]) between the spinor fields propagating in the bulk space and the QCD interpolating operators creating the nucleons on the boundary of AdS space. The appropriate boundary conditions for the bulk field on the boundary of AdS space ensure that such correspondence is precise due to the equivalence of the functional integrals of both the boundary and bulk theories. In particular, in the boundary theory (QCD) we define the left- and right-handed chiral doublets of nucleons $\mathscr{O}_{L}=\left(p_{L}, n_{L}\right)^{T}$ and $\mathscr{O}_{R}=\left(p_{R}, n_{R}\right)^{T}$, which are fundamental representations of the chiral $S U_{L}(2)$ and $S U_{R}(2)$ subgroups. Since the chiral symmetry of the boundary theory is equivalent to the gauge symmetry in the bulk, we need to introduce the pair of bulk fermion fields $\Psi_{ \pm}(x, z)$, which are holographic analogues of the $\mathscr{O}_{R / L}$ operators. In particular, the bulk fields $\Psi_{ \pm}(x, z)$ contain important information about the baryon structure. On one side, their boundary values (non-normalizable solutions) are analogues of the sources for the QCD interpolating operators, which then via the evaluation of the Euclidean generating functionals produce the correlation functions of QCD operators. On the other side, these fields contain normalizable modes (these are 
regular and therefore are vanishing on the boundary) - profiles in extra dimension, which correspond to the baryon wave functions or expectation values of QCD operators. In our approach the conformal and chiral symmetries are spontaneously broken via the introduction of the background field (dilaton) $\varphi(z)$ in the effective action. We choose the quadratic dependence of the dilaton on the holographic coordinate $z$, i.e. $\varphi(z)=\kappa^{2} z^{2}$ with $\kappa$ being a free scale parameter, which scales as $\mathscr{O}\left(\sqrt{N_{c}}\right)$ in the large $N_{c}$-expansion. In particular, later we show that the nucleon (baryon) mass is proportional to the parameter $\kappa$, which is consistent with large $N_{c}$ QCD: $M_{N} \sim \kappa \sqrt{N_{c}} \sim N_{c}$. The main advantage of the dilaton with quadratic profile is the possibility to produce linear Regge-like trajectories for hadron masses.

An additional source for the breaking of chiral and conformal symmetries is the coupling of the $\Psi_{+}(x, z)$ and $\Psi_{-}(x, z)$ fields, which is an essential basic block of hard-wall AdS/QCD approaches. In Ref. [36] we showed that in our dilaton-type model the contribution of $\Psi_{+}-\Psi_{-}$mixing coupling to the baryon masses is suppressed in comparison with the dilaton contribution by a factor $1 / N_{c}$.

In latter models the conformal invariance is broken by the introduction of an infrared brane, cutting the AdS geometry in the $z$-direction. In addition the couplings of the $\Psi_{+}(x, z)$ and $\Psi_{-}(x, z)$ fields with the scalar bulk field of dimension-3 are included. Due to the existence of a VEV of this scalar field, the chiral and conformal invariances are broken. In our approach such a mechanism could in principle be included, however it is a higher-order effect since it is generated by operators of dimension higher than 2. In particular, an extra power of the holographic coordinate gives the extra power of $1 / \kappa$, which scales as $1 / \sqrt{N_{c}}$.

The relevant AdS/QCD action for the description of the nucleon electromagnetic and axial form factors is constructed in terms of the fermion fields $\Psi_{ \pm, \tau}(x, z)$ with spin $J=1 / 2$ and scaling dimension $\tau$ (the isospin index corresponding to the proton and neutron components is suppressed for simplicity), the vector field $V_{M}(x, z)$ with spin $J=1$ (holographic analogue of the electromagnetic field) and the axial field $A_{M}(x, z)$ (holographic analogue of the axial isovector field) [18, 20, 22, 24]:

$$
\begin{aligned}
S & =\int d^{4} x d z \sqrt{g} e^{-\varphi(z)}\left\{\mathscr{L}_{\Psi}(x, z)+\mathscr{L}_{V+A}(x, z)+\mathscr{L}_{\text {int }}(x, z)\right\}, \\
\mathscr{L}_{\Psi}(x, z) & =\sum_{i=+,-} \sum_{\tau} c_{\tau} \bar{\Psi}_{i, \tau}(x, z) \hat{\mathscr{D}}_{i}(z) \Psi_{i, \tau}(x, z), \\
\mathscr{L}_{V+A}(x, z) & =-\frac{1}{4} V_{M N}(x, z) V^{M N}(x, z)-\frac{1}{4} A_{M N}(x, z) A^{M N}(x, z), \\
\mathscr{L}_{\text {int }}(x, z) & =\sum_{i=+,-} \sum_{\tau} c_{\tau} \bar{\Psi}_{i, \tau}(x, z)\left\{\hat{\mathscr{V}}_{i}(x, z)+\hat{\mathscr{A}}_{i}(x, z)\right\} \Psi_{i, \tau}(x, z),
\end{aligned}
$$

where

$$
\begin{aligned}
\hat{\mathscr{D}}_{ \pm}(z) & =\frac{i}{2} \Gamma^{M} \stackrel{\leftrightarrow}{\partial}_{M} \mp\left(\mu+U_{F}(z)\right), \\
\hat{\mathscr{V}}_{ \pm}(x, z) & =Q_{N} \Gamma^{M} V_{M}(x, z) \pm \frac{i}{4} \eta_{V}\left[\Gamma^{M}, \Gamma^{N}\right] V_{M N}(x, z) \pm g_{V} \tau_{3} \Gamma^{M} i \Gamma^{z} V_{M}(x, z), \\
\hat{\mathscr{A}}_{ \pm}(x, z) & =\frac{\tau_{3}}{2}\left(\mp \Gamma^{M} A_{M}(x, z)+\frac{i}{4} \eta_{A}\left[\Gamma^{M}, \Gamma^{N}\right] A_{M N}(x, z)+g_{A} \Gamma^{M} i \Gamma^{z} A_{M}(x, z)\right) .
\end{aligned}
$$

Here $F_{M N}=\partial_{M} F_{N}-\partial_{N} F_{M}(F=V, A)$ is the stress tensor of the vector (axial) field, $Q_{N}=\operatorname{diag}(1,0)$ is the nucleon charge matrix, $\tau_{3}=\operatorname{diag}(1,-1)$ is the Pauli isospin matrix, $A \stackrel{\leftrightarrow}{\partial} B \equiv A(\partial B)-(\partial A) B$, 
$\varphi(z)=\kappa^{2} z^{2}$ is the dilaton field with $\kappa$ being a free scale parameter. $\Gamma^{M}=\varepsilon_{a}^{M} \Gamma^{a}$ and $\Gamma^{a}=\left(\gamma^{\mu},-i \gamma^{5}\right)$ are the five-dimensional Dirac matrices (we use the chiral representation for the $\gamma^{\mu}$ and $\gamma^{5}$ matrices). Note that the non-abelian part of the action is irrelevant for the results predicted in the paper: the mass spectrum, the electromagnetic and axial isovector form factors of nucleons. The quantity $\mu$ is the bulk fermion mass related to the scaling dimension $\tau$ as $m=\mu R=\tau-3 / 2$. Notice that the scaling dimension of the AdS fermion field is holographically identified with the scaling dimension of the baryon interpolating operator $\tau=N+L$, where $N$ is the number of partons in the baryon and $L=\max \left|L_{z}\right|$ is the maximal value of the $z$-component of the quark orbital angular momentum in the light-front wavefunction [4, 7]. In the following we restrict to the ground state of nucleons with $L=0 . U_{F}(z)=\varphi(z) / R$ is the dilaton field dependent effective potential. Its presence is necessary due to the following reason. The form of the potential $U_{F}(z)$ is constrained in order to get solutions of the EOMs for the fermionic Kaluza-Klein (KK) modes of left and right chirality, and to have the correct asymptotics of the nucleon electromagnetic form factors at large $Q^{2}$ [18, 20, 22].

Notice that the fermion masses $m$ and effective potentials $U_{F}(z)$ corresponding to the fields $\Psi_{+}$ and $\Psi_{-}$have opposite signs according to the $P$-parity transformation In particular, the absolute sign of the fermion mass is related to the chirality of the boundary operator [27, 28]. According to our conventions the QCD operators $\mathscr{O}_{R}$ and $\mathscr{O}_{L}$ have positive and negative chirality, and therefore the mass terms of the bulk fields $\Psi_{+}$and $\Psi_{-}$have absolute signs "plus" and "minus", respectively. In addition to the minimal coupling of the fermion with the vector and the axial fields, we also include other possible (nonminimal) couplings. In particular we introduce: 1) a nonminimal coupling of fermion and vector fields in order to generate the Pauli form factors of the nucleon; 2) a minimaltype coupling, which is absent in four dimensions, but exists in five dimensions.

We will show that these terms do not renormalize the electric charge of the bulk fields and contribute only to the $Q^{2}$-dependence or to the slopes of the Dirac nucleon form factors. The coupling $g_{V}$ is a free parameter which is not constrained by gauge invariance or discrete symmetries $(P-, C-$ or $T$-parity conservation). We will fix these terms by improving the description of the electromagnetic nucleon radii. The diagonal matrix $\eta_{V}=\operatorname{diag}\left\{\eta_{V}^{p}, \eta_{V}^{n}\right\}$ contains the coupling constrained by the anomalous magnetic moments $k_{p, n}$ of the nucleons $\left(k_{p}=1.793\right.$ and $k_{n}=-1.913$ are given in units of nucleon magnetons or n.m.) as $\eta_{V}^{p, n} \sim k_{p, n} \cdot \kappa / m_{N}$ where $m_{N}$ is the nucleon mass.

In the case of the axial field we additionally include: 1) the nonminimal coupling of fermion and axial fields, which does not renormalize the axial charge, but gives a nontrivial contribution to the $Q^{2}$-dependence and to the slope of the corresponding axial isovector form factor of the nucleon; 2) the axial-type coupling proportional to the nucleon charge, which defines the leading contribution to the isovector axial form factor of the nucleon.

The fields $\Psi_{\tau}$ describe the AdS fermion field with different scaling dimension $\tau$, which in the large $N_{c}$ expansion scales as $\tau \sim N_{c}$. Restricting to a finite number of colors $N_{c}=3$, we use $\tau=3,4,5$, etc. In this paper we restrict to the three leading contributions $\tau=3,4$ and 5. According to the AdS/QCD dictionary the fermion field $\Psi_{\tau=3}$ is the holographic analogue of the nucleon interpolating operator with twist-dimension 3, which means that the corresponding nucleon Fock state contains 3 valence quarks. The fermion field $\Psi_{\tau=4}$ effectively models the nucleon operator with twist-4 (the corresponding Fock state contains 4 partons -3 valence quarks plus a gluon field). Finally, the fermion field $\Psi_{\tau=5}$ models the nucleon operators with twist-5. The corresponding Fock states contain 5 partons: (1) 3 valence quarks plus a $q \bar{q}$ pair of sea quarks or (2) 3 valence quarks 
plus 2 gluons. Therefore, the coefficients $c_{\tau}$ are a set of parameters which take into account the mixing of AdS fermion fields with different scaling dimension $\tau$. The set of mixing parameters $c_{\tau}$ is constrained by the correct normalization of the kinetic term of the four-dimensional spinor field and by charge conservation as $\sum_{\tau} c_{\tau}=1$ (see details below). In the consideration of the vector (axial) field we apply the axial gauge $V(A)_{z}(x, z)=0$.

In Figs. 1-3 we give an illustration for the inclusion of twist-3 (Fig.1), twist-4 (Fig.2) and twist-5 (Fig.3) partonic Fock states in the description of electromagnetic transition between nucleons. Due do the gauge/gravity duality we identify the respective sets of QCD diagrams to the corresponding vector-current transition matrix elements involving the fermion field of corresponding twist-dimension. One should stress that AdS/QCD gives a unique possibility to describe a set of QCD diagrams just by one graph (for each partonic content of the nucleon) and obtain predictions for hadronic observables in analytical form.

Finalizing our discussion of the 5D effective action (2.2) we would like to point out again that it obeys $P-, C$ - and $T$-invariance. This action further contains new terms describing the interaction of vector and axial fields with fermions, which were not considered before in the context of AdS/QCD. These new terms do not renormalize the charge (i.e. vanish at $Q^{2}=0$ ), but they contribute to the $Q^{2}$-dependence and the slopes of the corresponding form factors. Their relevance for giving a sufficient description of the data will be shown further on.

\section{Mass spectrum}

One advantage of the soft-wall AdS/QCD model is that most of the calculations can be done analytically. In a first step, we show how in this approach the baryon spectrum and wave functions are generated. We follow the procedure pursued in Refs. [18, 20, 22, 24,. Dropping the vector and axial fields, and rescaling the fermionic fields as $\Psi_{i, \tau}(x, z)=e^{\varphi(z) / 2} \psi_{i, \tau}(x, z)$ we remove the dilaton field from the overall exponential. In terms of the field $\psi_{\tau}(x, z)$ the modified action in the Lorentzian signature reads as

$$
S_{0}=\int d^{4} x d z e^{4 A} \sum_{i=+,-} \sum_{\tau} c_{\tau} \bar{\psi}_{i, \tau}(x, z)\left\{i \not \partial+\gamma^{5} \partial_{z}+2 A^{\prime} \gamma^{5}-\delta_{i} \frac{e^{A}}{R}(m+\varphi)\right\} \psi_{i, \tau}(x, z),
$$

where $\partial=\gamma^{\mu} \partial_{\mu}, \delta_{ \pm}= \pm 1$ and the fermion field $\psi_{i, \tau}(x, z)$ satisfies the following equation of motion (EOM) [18, 20, 22, 24]:

$$
\left[i \not \partial+\gamma^{5} \partial_{z}+2 A^{\prime}(z) \gamma^{5} \mp \frac{e^{A(z)}}{R}(m+\varphi(z))\right] \psi_{ \pm, \tau}(x, z)=0 .
$$

Based on these solutions the fermionic action should be extended by an extra term in the ultraviolet boundary (see details in Refs. [20, 32]) in order to guarantee the gauge/gravity correspondence - equivalence between the AdS functional integral and the generating functional for correlation functions in QCD.

Next we split the fermion field into left- and right-chirality components

$$
\psi_{i, \tau}(x, z)=\psi_{i, \tau}^{L}(x, z)+\psi_{i, \tau}^{R}(x, z), \quad \psi_{i, \tau}^{L / R}(x, z)=\frac{1 \mp \gamma^{5}}{2} \psi_{i, \tau}(x, z) .
$$


and perform a KK expansion for the $\psi_{i, \tau}^{L / R}(x, z)$ fields:

$$
\psi_{i, \tau}^{L / R}(x, z)=\frac{1}{\sqrt{2}} \sum_{n} \psi_{n}^{L / R}(x) F_{i, \tau, n}^{L / R}(z)
$$

where $\psi_{n}^{L / R}(x)$ are the four-dimensional boundary fields (KK modes). These are Weyl spinors forming the Dirac bispinors $\psi_{n}(x)=\psi_{n}^{L}(x)+\psi_{n}^{R}(x)$, and $F_{i, \tau, n}^{L / R}(z)$ are the normalizable profile functions. Due to four-dimensional $P$ - and $C$-parity invariance the bulk profiles are related as: $F_{ \pm, \tau, n}^{R}(z)=\mp F_{\mp, \tau, n}^{L}(z)$. Using this constraint in the following we use the simplified notations:

$$
F_{\tau, n}^{R}(z) \equiv F_{+, \tau, n}^{R}(z)=-F_{-, \tau, n}^{L}(z), \quad F_{\tau, n}^{L}(z) \equiv F_{+, \tau, n}^{L}(z)=F_{-, \tau, n}^{R}(z) .
$$

Note that the profiles $F_{\tau, n}^{L / R}(z)$ are the holographic analogues of the nucleon wave functions with specific radial quantum number $n$ and twist dimension $\tau$ (the latter corresponds to the specific partonic content of the nucleon Fock component), which satisfy the two coupled one-dimensional EOMs [18, 20, 22]:

$$
\left[\partial_{z} \pm \frac{e^{A}}{R}(m+\varphi)+2 A^{\prime}\right] F_{\tau, n}^{L / R}(z)= \pm M_{n \tau} F_{\tau, n}^{R / L}(z) .
$$

Therefore, our main idea is to find the solutions for the bulk profiles of the AdS field in the $z-$ direction, and then calculate the physical properties of hadrons. Doing the substitution $F_{\tau, n}^{L / R}(z)=$ $e^{-2 A(z)} f_{\tau, n}^{L / R}(z)$. After straightforward algebra one can obtain the decoupled Schrödinger-type EOMs:

$$
\left[-\partial_{z}^{2}+\kappa^{4} z^{2}+2 \kappa^{2}\left(m \mp \frac{1}{2}\right)+\frac{m(m \pm 1)}{z^{2}}\right] f_{\tau, n}^{L / R}(z)=M_{n \tau}^{2} f_{\tau, n}^{L / R}(z)
$$

where

$$
\begin{aligned}
& f_{\tau, n}^{L}(z)=\sqrt{\frac{2 \Gamma(n+1)}{\Gamma(n+\tau)}} \kappa^{\tau} z^{\tau-1 / 2} e^{-\kappa^{2} z^{2} / 2} L_{n}^{\tau-1}\left(\kappa^{2} z^{2}\right) \\
& f_{\tau, n}^{R}(z)=\sqrt{\frac{2 \Gamma(n+1)}{\Gamma(n+\tau-1)}} \kappa^{\tau-1} z^{\tau-3 / 2} e^{-\kappa^{2} z^{2} / 2} L_{n}^{\tau-2}\left(\kappa^{2} z^{2}\right)
\end{aligned}
$$

and

$$
M_{n \tau}^{2}=4 \kappa^{2}(n+\tau-1) .
$$

In above formulas we substituted $m=\tau-3 / 2$.

One can see that the functions $F_{\tau, n}^{L / R}(z)=e^{-2 A(z)} f_{\tau, n}^{L / R}(z)$ have the correct scaling behavior for small $z: F_{\tau, n}^{L}(z) \sim z^{\tau+3 / 2}, F_{\tau, n}^{R}(z) \sim z^{\tau+1 / 2}$, when identified with the corresponding nucleon wave functions with twist $\tau$, and vanish at large $z$ (confinement). Below, in the discussion of the electromagnetic properties of the nucleon, we explicitly demonstrate that the Dirac and Pauli form factors have the correct scaling dependence at large $Q^{2}$.

The nucleon mass is identified with the expression

$$
M_{n}=\sum_{\tau} c_{\tau} M_{n \tau}=2 \kappa \sum_{\tau} c_{\tau} \sqrt{n+\tau-1}
$$


Due to the dilaton the chiral and conformal symmetries are spontaneously broken in our approach. Switching off the dilaton field (which corresponds to the limit $\kappa=0$ ) leads to the restoration of the chiral and conformal symmetries. In particular, the masses of the bulk profiles $f_{\tau, n}^{L / R}(z)$ and the nucleon mass vanish in this limit. As we stressed before, the nucleon (baryon) mass is proportional to the parameter $\kappa$ and this is consistent with large $N_{c}$ QCD: $M_{N} \sim \kappa \sqrt{\tau} \sim N_{c}$, where $\tau \sim N_{c}$.

Integration over the holographic coordinate $z$, with the use of the normalization condition for the profile functions $f_{\tau, n}^{L / R}(z)$, gives a four-dimensional action for the fermion field $\psi_{n}(x)=$ $\psi_{n}^{L}(x)+\psi_{n}^{R}(x)$ :

$$
S_{0}=\sum_{n} \int d^{4} x \bar{\psi}_{n}(x)\left[i \not \partial-M_{n}\right] \psi_{n}(x) .
$$

This last equation is a manifestation of the gauge-gravity duality. It explicitly demonstrates that effective actions for conventional hadrons in 4 dimensions can be generated from actions for bulk fields propagating in 5 dimensional AdS space. The effect of the extra-dimension is encoded in the hadronic mass squared (in our case in the nucleon mass $M_{n}$, where $n$ is the radial quantum number), which is the superposition of the solutions of the Schrödinger equation for the KK profiles in the extra dimension. Notice that the constraint $\sum_{\tau} c_{\tau}=1$ for the mixing parameters $c_{\tau}$ was essential in order to get the correct normalization of the kinetic term $\bar{\psi}_{n}(x) i \not \partial \psi_{n}(x)$ of the four-dimensional spinor field.

\section{Electroweak structure of nucleons}

In this section we present the related numerical analysis of nucleon properties: magnetic moments $\left(\mu_{p}=1+\kappa_{p}, \mu_{n}=\kappa_{n}\right.$ ), electromagnetic and axial radii $\left(r_{E}^{p},\left\langle r_{E}^{2}\right\rangle^{n}, r_{M}^{p}, r_{M}^{n}, r_{A}\right)$, isovector axial and electromagnetic Dirac, Pauli and Sachs form factors and their ratios in the Euclidean region (see definitions in [36). The nucleon electromagnetic form factors $F_{1}^{N}$ and $F_{2}^{N}(N=p, n)$ correspond to proton and neutron) are conventionally defined by the matrix element of the electromagnetic current as

$$
\left\langle p^{\prime}\left|J^{\mu}(0)\right| p\right\rangle=\bar{u}\left(p^{\prime}\right)\left[\gamma^{\mu} F_{1}^{N}(t)+\frac{i}{2 m_{N}} \sigma^{\mu v} q_{v} F_{2}^{N}(t)\right] u(p),
$$

where $q=p^{\prime}-p$ is the momentum transfer and $t=q^{2} ; m_{N}$ is the nucleon mass; and $F_{1}^{N}$ and $F_{2}^{N}$ are the Dirac and Pauli form factors, which are normalized to the electric charge $e_{N}$ and anomalous magnetic moment $k_{N}$ of the corresponding nucleon: $F_{1}^{N}(0)=e_{N}$ and $F_{2}^{N}(0)=k_{N}$.

The nucleon isovector axial form factor $G_{A}(t)$ is conventionally defined by the matrix element of the axial isovector current as

$$
\left\langle p^{\prime}\left|A_{3}^{\mu}(0)\right| p\right\rangle=\bar{u}\left(p^{\prime}\right)\left[\gamma^{\mu} G_{A}(t)+\frac{q^{\mu}}{2 m_{N}} G_{P}(t)\right] \gamma^{5} \frac{\tau_{3}}{2} u(p),
$$

which is normalized to the nucleon axial charge $G_{A}(0)=g_{A}$.

In our approach the nucleon electromagnetic and axial form factors are generated respectively by the actions

$$
S_{\text {int }}^{V, A}=\int d^{4} x d z \sqrt{g} e^{-\varphi(z)} \mathscr{L}_{\text {int }}^{V, A}(x, z)
$$


containing the minimal and nonminimal couplings of fermion and vector AdS fields. The expressions for the nucleon form factors are given in [36].

The five free parameters $\kappa, c_{3}, c_{4}, g_{V}$ and $\eta_{A}$ are fixed to the values $\kappa=383 \mathrm{MeV}, c_{3}=1.25$, $c_{4}=0.16, g_{V}=0.3, \eta_{A}=0.5$. Note that the parameter $c_{5}$ is expressed through $c_{3}$ and $c_{4}$ as $c_{5}=1-c_{3}-c_{4}=-0.41$. Here the parameters $c_{3}, c_{4}$ are constrained by the nucleon mass. The parameter $\kappa$ is fixed by the nucleon mass and the electromagnetic radii. The parameters $g_{V}$ and $\eta_{A}$ are fitted by fine tuning of the neutron electromagnetic and nucleon axial radius, respectively. Notice also that the other parameters are fixed by the magnetic moments and the axial charge of nucleons and should not be counted as free parameters: $g_{A}=1.270, \eta_{V}^{p}=\frac{\kappa\left(\mu_{p}-1\right)}{2 m_{N} C_{0}}=0.30$, $\eta_{V}^{n}=\frac{\kappa \mu_{n}}{2 m_{N} C_{0}}=-0.32, C_{0}=\sqrt{2} c_{3}+\sqrt{3} c_{4}+2 c_{5}$. In Table I we present the results for the nucleon mass and the electroweak properties of nucleons. Results for the nucleon electromagnetic form factors in comparison to known data are shown in Figs. 4-6. In particular, we present the predictions for the ratios of proton charge and nucleon magnetic form factors to the dipole form factor $G_{D}$. ratio $\mu_{p} G_{E}^{p}\left(Q^{2}\right) / G_{M}^{p}\left(Q^{2}\right)$, for the charge neutron form factor and the ratio $G_{E}^{n}\left(Q^{2}\right) / G_{M}^{n}\left(Q^{2}\right)$, for the Dirac nucleon form factors multiplied by $Q^{4}$, the ratios of the Pauli and Dirac form factors of the proton multiplied with $Q^{2}$ and with $Q^{2} / \log ^{2}\left(Q^{2} / \Lambda^{2}\right)$, where $\Lambda=0.3 \mathrm{GeV}$ and for the ratio of nucleon axial isovector form factor to the dipole form factor $G_{A}^{D}\left(Q^{2}\right)$.

We demonstrated that the soft-wall holographic model in the semiclassical approximation reproduces the main features of the electromagnetic structure of the nucleon. In particular, we achieved the following results: the analytical power scaling of the elastic nucleon form factors at large momentum transfers in accordance with quark-counting rules; reproduction of experimental data for magnetic moments and electromagnetic radii. One can see that with a minimal number of free parameters (five parameters) we obtain a reasonable description of the nucleon electromagnetic and axial-vector form factors including the correct power scaling at large $Q^{2}$. It demonstrates that the soft-wall model successfully describes nucleon structure at any resolution scale. In a next step, one can include effects of quark masses and extend the approach to nucleon resonances, light baryons with higher spins, strange and heavy baryons.

\section{Acknowledgments}

The authors thank Stan Brodsky, Guy de Téramond and Werner Vogelsang for useful discussions. This work was supported by the DFG under Contract No. LY 114/2-1, by Federal Targeted Program "Scientific and scientific-pedagogical personnel of innovative Russia" Contract No. 02.740.11.0238, by FONDECYT (Chile) under Grant No. 1100287. V. E. L. would like to thank Departamento de Física y Centro Científico Tecnológico de Valparaíso (CCTVal), Universidad Técnica Federico Santa María, Valparaíso, Chile for warm hospitality. A. V. acknowledges the financial support from FONDECYT (Chile) Grant No. 3100028. 


\section{References}

[1] J. M. Maldacena, Adv. Theor. Math. Phys. 2, 231 (1998) [Int. J. Theor. Phys. 38, 1113 (1999)] [arXiv:hep-th/9711200]; S. S. Gubser, I. R. Klebanov and A. M. Polyakov, Phys. Lett. B 428, 105 (1998) [arXiv:hep-th/9802109]; E. Witten, Adv. Theor. Math. Phys. 2, 253 (1998) [arXiv:hep-th/9802150].

[2] Y. Kim and D. Yi, Adv. High Energy Phys. 2011, 1 (2011) [arXiv:1107.0155 [hep-ph]].

[3] A. Karch, E. Katz, D. T. Son and M. A. Stephanov, Phys. Rev. D 74, 015005 (2006) [arXiv:hep-ph/0602229].

[4] S. J. Brodsky, G. F. de Teramond, Phys. Rev. Lett. 96, 201601 (2006) [hep-ph/0602252].

[5] S. J. Brodsky and G. F. de Teramond, Phys. Rev. D 77, 056007 (2008) [arXiv:0707.3859 [hep-ph]].

[6] S. J. Brodsky, G. F. de Teramond and A. Deur, Phys. Rev. D 81, 096010 (2010) [arXiv:1002.3948 [hep-ph]].

[7] G. F. de Teramond and S. J. Brodsky, AIP Conf. Proc. 1296, 128 (2010) [arXiv:1006.2431 [hep-ph]].

[8] O. Andreev, Phys. Rev. D 73, 107901 (2006) [arXiv:hep-th/0603170]; O. Andreev and V. I. Zakharov, Phys. Rev. D 74, 025023 (2006) [arXiv:hep-ph/0604204].

[9] H. Forkel, M. Beyer and T. Frederico, JHEP 0707, 077 (2007) [arXiv:0705.1857 [hep-ph]]; Int. J. Mod. Phys. E 16, 2794 (2007) [arXiv:0705.4115 [hep-ph]]; W. de Paula, T. Frederico, H. Forkel and M. Beyer, Phys. Rev. D 79, 075019 (2009) [arXiv:0806.3830 [hep-ph]].

[10] B. Galow, E. Megias, J. Nian and H. J. Pirner, Nucl. Phys. B 834, 330 (2010) [arXiv:0911.0627 [hep-ph]]; J. Nian and H. J. Pirner, Nucl. Phys. A 833, 119 (2010) [arXiv:0908.1330 [hep-ph]].

[11] H. R. Grigoryan and A. V. Radyushkin, Phys. Rev. D 76, 095007 (2007) [arXiv:0706.1543 [hep-ph]].

[12] H. Forkel, Phys. Rev. D 78, 025001 (2008) [arXiv:0711.1179 [hep-ph]]; H. J. Kwee and R. F. Lebed, Phys. Rev. D 77, 115007 (2008) [arXiv:0712.1811 [hep-ph]]. P. Colangelo, F. De Fazio, F. Jugeau and S. Nicotri, Phys. Lett. B 652, 73 (2007) [arXiv:hep-ph/0703316];

[13] T. Gherghetta, J. I. Kapusta and T. M. Kelley, Phys. Rev. D 79, 076003 (2009) [arXiv:0902.1998 [hep-ph]]; T. M. Kelley, S. P. Bartz and J. I. Kapusta, Phys. Rev. D 83, 016002 (2011) [arXiv:1009.3009 [hep-ph]]; Y. Q. Sui, Y. L. Wu, Z. F. Xie and Y. B. Yang, Phys. Rev. D 81, 014024 (2010) [arXiv:0909.3887 [hep-ph]]; M. Fujita, K. Fukushima, T. Misumi and M. Murata, Phys. Rev. D 80, 035001 (2009) [arXiv:0903.2316 [hep-ph]]; S. S. Afonin, Int. J. Mod. Phys. A 25, 5683 (2010). [arXiv:1001.3105 [hep-ph]]; C. Marquet, C. Roiesnel and S. Wallon, JHEP 1004, 051 (2010) [arXiv:1002.0566 [hep-ph]]; H. R. Grigoryan, P. M. Hohler and M. A. Stephanov, Phys. Rev. D 82, 026005 (2010) [arXiv:1003.1138 [hep-ph].

[14] A. Vega and I. Schmidt, Phys. Rev. D 78, 017703 (2008) [arXiv:0806.2267 [hep-ph]].

[15] A. Vega and I. Schmidt, Phys. Rev. D 79, 055003 (2009) [arXiv:0811.4638 [hep-ph]].

[16] A. Vega, I. Schmidt, T. Branz, T. Gutsche and V. E. Lyubovitskij, Phys. Rev. D 80, 055014 (2009) [arXiv:0906.1220 [hep-ph]].

[17] A. Vega, I. Schmidt, Phys. Rev. D 82, 115023 (2010) [arXiv:1005.3000 [hep-ph]].

[18] G. F. de Teramond and S. J. Brodsky, Nucl. Phys. Proc. Suppl. 199, 89 (2010) [arXiv:0909.3900 [hep-ph]]; S. J. Brodsky and G. F. de Teramond, arXiv:0802.0514 [hep-ph]; AIP Conf. Proc. 1257, 59 (2010) [arXiv:1001.5193 [hep-ph]]; AIP Conf. Proc. 1432, 168 (2012) [arXiv:1108.0965 [hep-ph]]. S. J. Brodsky and G. F. de Teramond, AIP Conf. Proc. 1388, 22 (2011) [arXiv:1103.1186 [hep-ph]]. 
[19] G. F. de Teramond and S. J. Brodsky, [arXiv:1203.4025 [hep-ph]].

[20] Z. Abidin and C. E. Carlson, Phys. Rev. D 79, 115003 (2009) [arXiv:0903.4818 [hep-ph]].

[21] T. Branz, T. Gutsche, V. E. Lyubovitskij, I. Schmidt, A. Vega, Phys. Rev. D 82, 074022 (2010) [arXiv:1008.0268 [hep-ph]].

[22] A. Vega, I. Schmidt, T. Gutsche, V. E. Lyubovitskij, Phys. Rev. D 83, 036001 (2011) [arXiv:1010.2815 [hep-ph]].

[23] A. Karch, E. Katz, D. T. Son, M. A. Stephanov, JHEP 1104, 066 (2011) [arXiv:1012.4813 [hep-ph]].

[24] T. Gutsche, V. E. Lyubovitskij, I. Schmidt and A. Vega, Phys. Rev. D 85, 076003 (2012) [arXiv:1108.0346 [hep-ph]].

[25] A. Vega, I. Schmidt, T. Gutsche and V. E. Lyubovitskij, Phys. Rev. D 85, 096004 (2012) [arXiv:1202.4806 [hep-ph]].

[26] G. F. de Teramond and S. J. Brodsky, Phys. Rev. Lett. 94, 201601 (2005) [arXiv:hep-th/0501022].

[27] D. K. Hong, T. Inami and H. U. Yee, Phys. Lett. B 646, 165 (2007) [arXiv:hep-ph/0609270].

[28] Y. Kim, C. H. Lee and H. U. Yee, Phys. Rev. D 77, 085030 (2008) [arXiv:0707.2637 [hep-ph]]; H. C. Ahn, D. K. Hong, C. Park and S. Siwach, Phys. Rev. D 80, 054001 (2009) [arXiv:0904.3731 [hep-ph]]; N. Maru and M. Tachibana, Eur. Phys. J. C 63, 123 (2009) [arXiv:0904.3816 [hep-ph]]; H. C. Kim, Y. Kim and U. Yakhshiev, JHEP 0911, 034 (2009) [arXiv:0908.3406 [hep-ph]]; P. Zhang, Phys. Rev. D 81, 114029 (2010) [arXiv:1002.4352 [hep-ph]]; Phys. Rev. D 82, 094013 (2010) [arXiv:1007.2163 [hep-ph]].

[29] A. Pomarol and A. Wulzer, Nucl. Phys. B 809, 347 (2009) [arXiv:0807.0316 [hep-ph]]; G. Panico and A. Wulzer, Nucl. Phys. A 825, 91 (2009) [arXiv:0811.2211 [hep-ph]].

[30] D. K. Hong, M. Rho, H. U. Yee and P. Yi, Phys. Rev. D 76, 061901 (2007) [arXiv:hep-th/0701276]; JHEP 0709, 063 (2007) [arXiv:0705.2632 [hep-th]].

[31] H. Hata, T. Sakai, S. Sugimoto and S. Yamato, Prog. Theor. Phys. 117, 1157 (2007) [arXiv:hep-th/0701280]; K. Hashimoto, T. Sakai and S. Sugimoto, Prog. Theor. Phys. 120, 1093 (2008) [arXiv:0806.3122 [hep-th]].

[32] M. Henningson and K. Sfetsos, Phys. Lett. B 431, 63 (1998) [arXiv:hep-th/9803251]; W. Mueck and K. S. Viswanathan, Phys. Rev. D 58, 106006 (1998) [arXiv:hep-th/9805145]; G. E. Arutyunov and S. A. Frolov, Nucl. Phys. B 544, 576 (1999) [arXiv:hep-th/9806216]; M. Henneaux, International Meeting on Mathematical Methods in Modern Theoretical Physics: School and Workshop, Tbilisi, Georgia, 1998, pp. 161-170; arXiv:hep-th/9902137; R. Contino and A. Pomarol, JHEP 0411, 058 (2004) [arXiv:hep-th/0406257].

[33] S. J. Brodsky, F. G. Cao and G. F. de Teramond, Phys. Rev. D 84, 075012 (2011) [arXiv:1105.3999 [hep-ph]].

[34] K. Nakamura et al. (Particle Data Group), J. Phys. G 37, 075021 (2010).

[35] S. J. Brodsky, arXiv:hep-ph/0208158.

[36] T. Gutsche, V. E. Lyubovitskij, I. Schmidt and A. Vega, Phys. Rev. D 86, 036007 (2012) [arXiv:1204.6612 [hep-ph]]. 
Table 1: Mass and electromagnetic properties of nucleons

\begin{tabular}{|c|c|c|}
\hline Quantity & Our results & Data [34] \\
\hline$m_{p}(\mathrm{GeV})$ & 0.93827 & 0.93827 \\
\hline$\mu_{p}$ (in n.m.) & 2.793 & 2.793 \\
\hline$\mu_{n}$ (in n.m.) & -1.913 & -1.913 \\
\hline$g_{A}$ & 1.270 & 1.2701 \\
\hline$r_{E}^{p}(\mathrm{fm})$ & 0.840 & $0.8768 \pm 0.0069$ \\
\hline$\left\langle r_{E}^{2}\right\rangle^{n}\left(\mathrm{fm}{ }^{2}\right)$ & -0.117 & $-0.1161 \pm 0.0022$ \\
\hline$r_{M}^{p}(\mathrm{fm})$ & 0.785 & $0.777 \pm 0.013 \pm 0.010$ \\
\hline$r_{M}^{n}(\mathrm{fm})$ & 0.792 & $0.862_{-0.008}^{+0.009}$ \\
\hline$r_{A}(\mathrm{fm})$ & 0.667 & $0.67 \pm 0.01$ \\
\hline
\end{tabular}




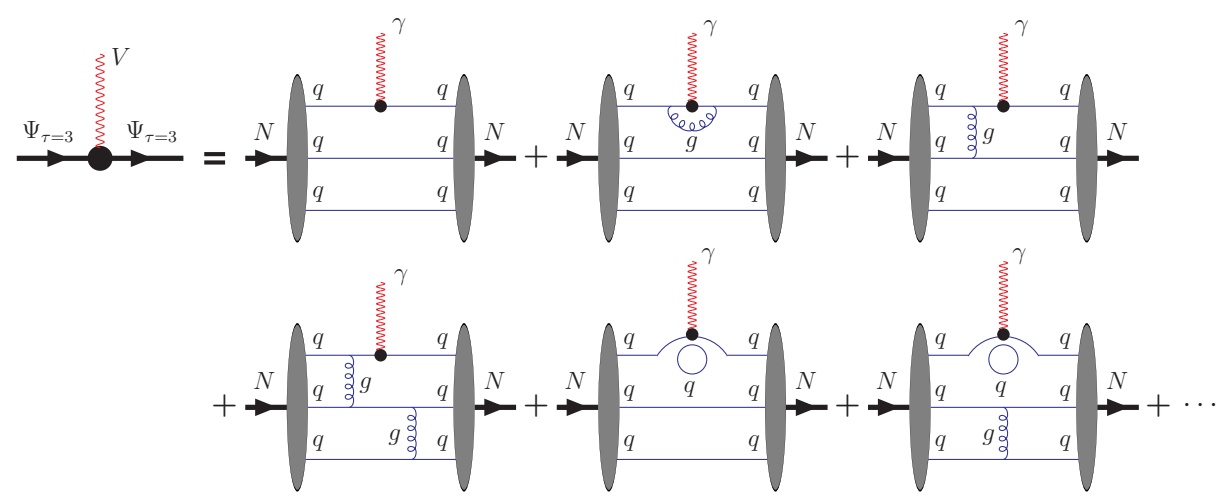

Figure 1: Gauge/gravity duality between the vector-current transition matrix element involving twist dimension-3 fermion fields in AdS and the electromagnetic matrix elements involving twist-3 partonic Fock states in nucleons.

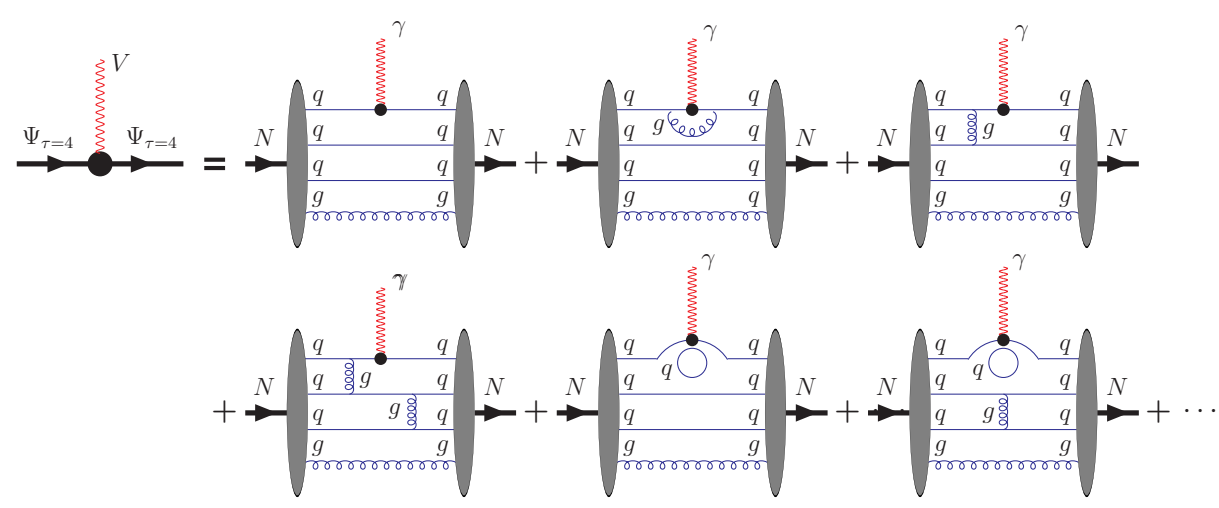

Figure 2: Gauge/gravity duality between the vector-current transition matrix element involving twist dimension-4 fermion fields in AdS and the electromagnetic matrix elements involving twist-4 partonic Fock states in nucleons.

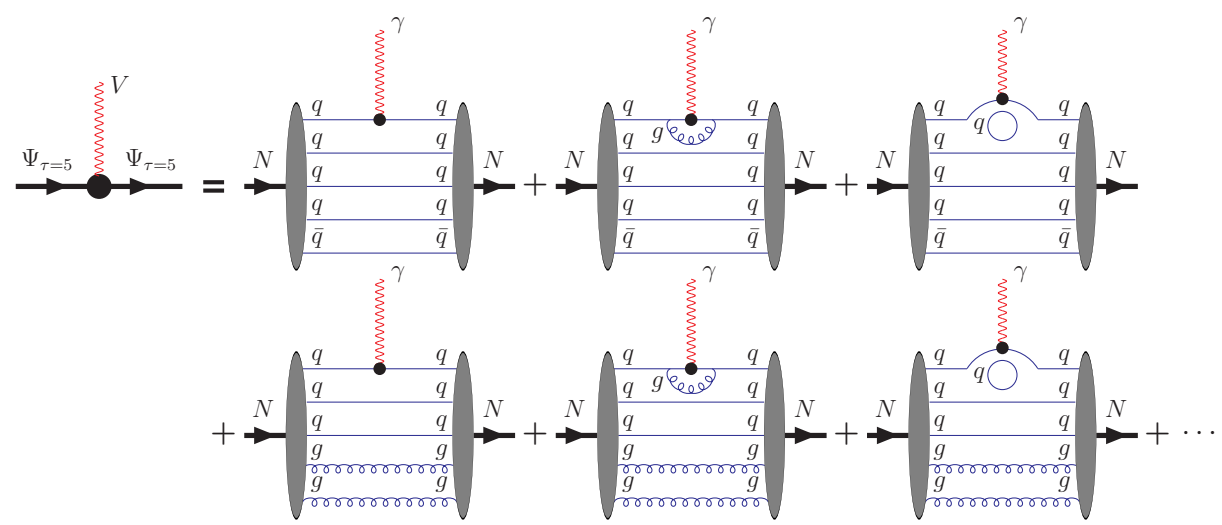

Figure 3: Gauge/gravity duality between the vector-current transition matrix element involving twist dimension-5 fermion fields in AdS and the electromagnetic matrix elements involving twist-5 partonic Fock states in nucleons. 

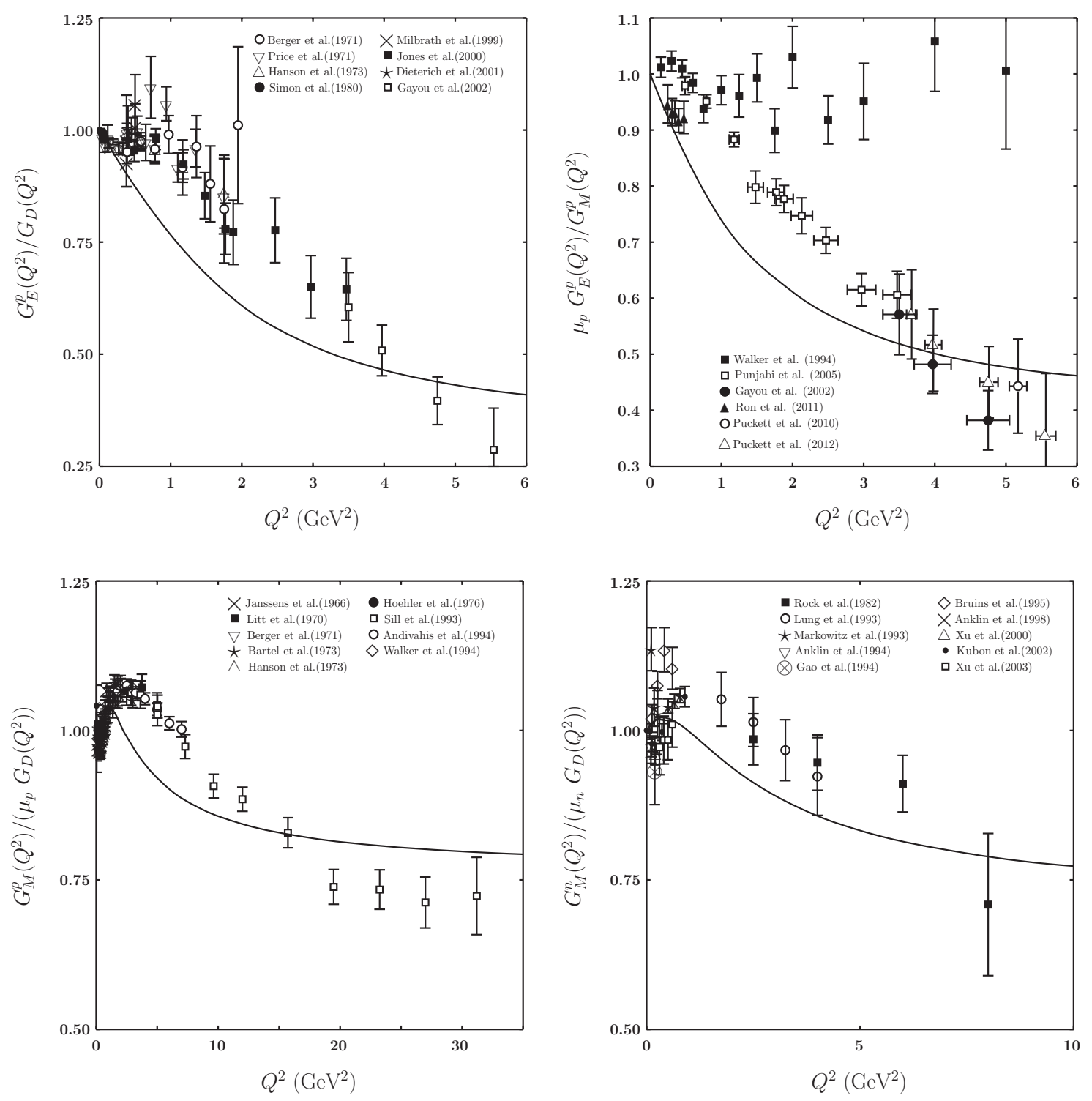

Figure 4: Ratios $G_{E}^{p}\left(Q^{2}\right) / G_{D}\left(Q^{2}\right), \mu_{p} G_{E}^{p}\left(Q^{2}\right) / G_{M}^{p}\left(Q^{2}\right), G_{M}^{p}\left(Q^{2}\right) /\left(\mu_{p} G_{D}\left(Q^{2}\right)\right)$ and $G_{M}^{n}\left(Q^{2}\right) /\left(\mu_{n} G_{D}\left(Q^{2}\right)\right)$. 

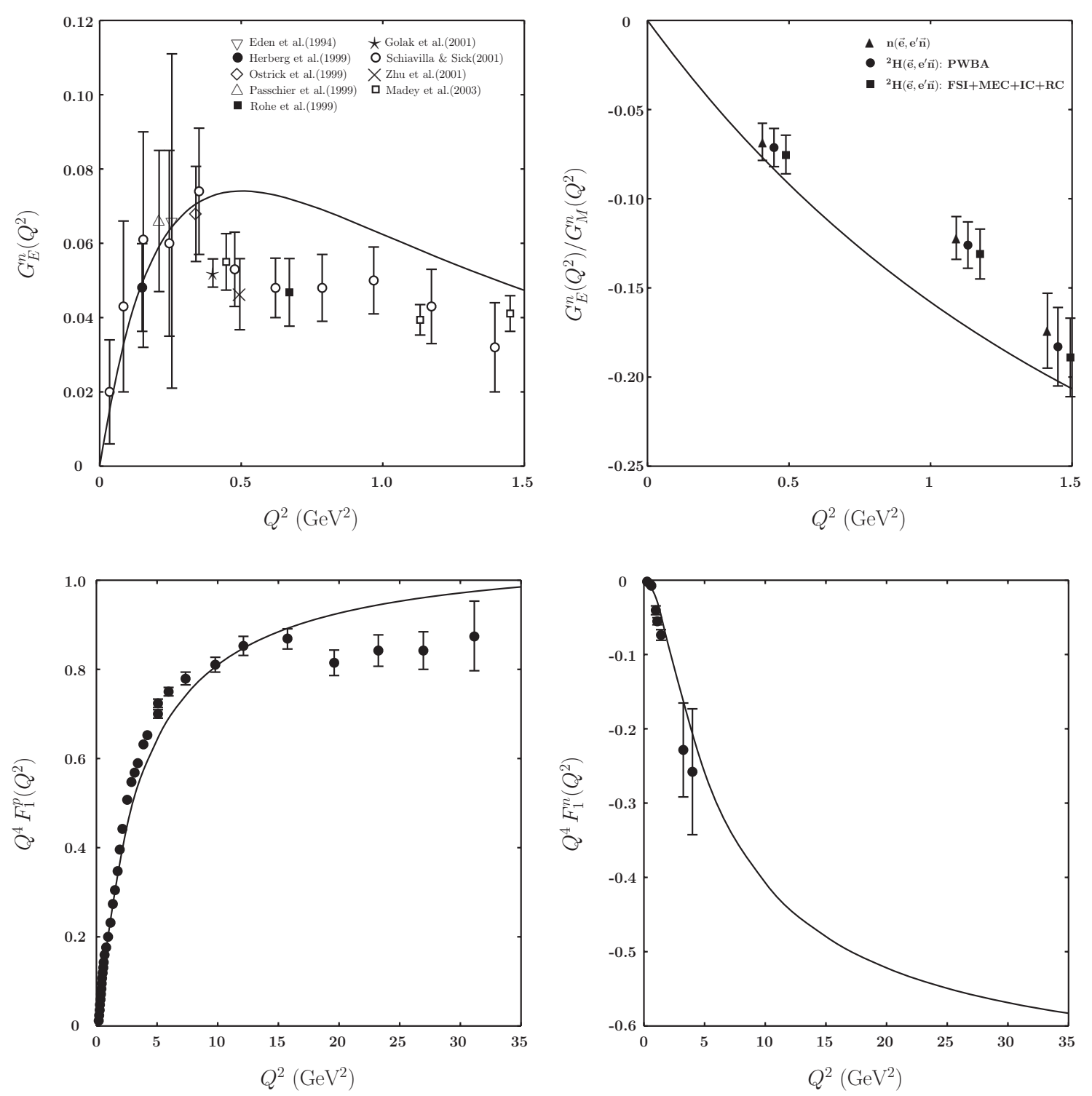

Figure 5: The charge neutron form factor, ratio $G_{E}^{n}\left(Q^{2}\right) / G_{M}^{n}\left(Q^{2}\right)$, proton and neutron Dirac form factor multiplied with $Q^{4}$. 

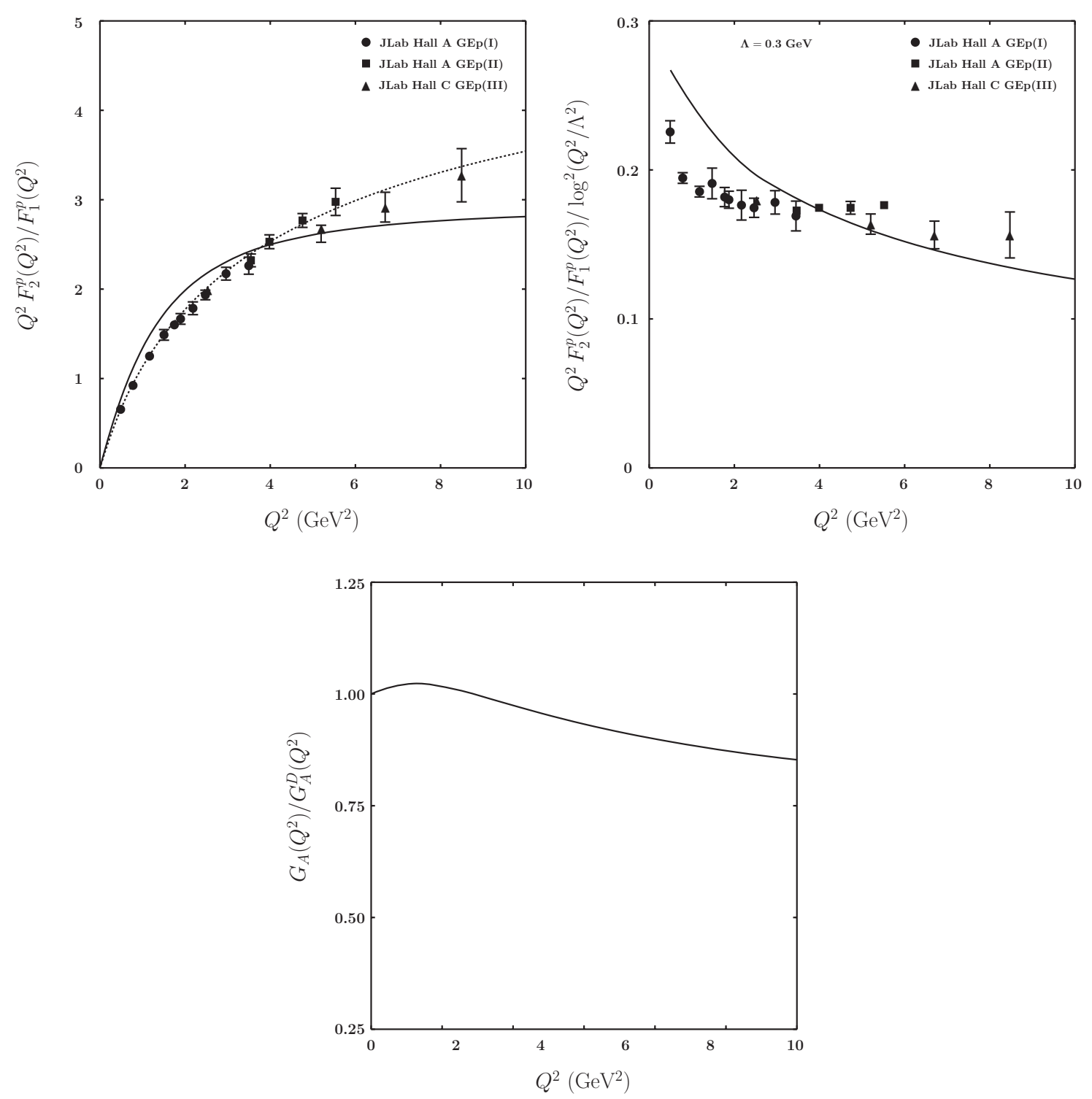

Figure 6: Ratios $Q^{2} F_{2}^{p}\left(Q^{2}\right) / F_{1}^{p}\left(Q^{2}\right), \quad Q^{2} F_{2}^{p}\left(Q^{2}\right) / F_{1}^{p}\left(Q^{2}\right) / \log ^{2}\left(Q^{2} / \Lambda^{2}\right)$ at $\Lambda=0.3 \mathrm{GeV}$ and $G_{A}\left(Q^{2}\right) / G_{A}^{D}\left(Q^{2}\right)$. 